\title{
Community-acquired Pneumonia Secondary to Legionella pneumophila and Streptococcus pneumoniae: A Rare Co-infection
}

\author{
Moeezullah Beg ${ }^{1}$, Hamza Arif ${ }^{2}$, Thomas Walsh ${ }^{3}$ \\ 1. Internal Medicine, University of Texas Health Science Center San Antonio, San Antonio, USA 2. Internal Medicine, \\ Allegheny Health Network, Pittsburgh, USA 3. Infectious Disease, Allegheny Health Network, Pittsburgh, USA
}

Corresponding author: Moeezullah Beg, beg@uthscsa.edu

\begin{abstract}
Community-acquired pneumonia (CAP) is a frequent cause of hospitalization in adults. Streptococcus pneumoniae is the most commonly identified pathogen in CAP whereas Legionella pneumophilia is infrequently identified in CAP. Although co-infections have been previously described, the presence of both pneumococcus and legionella together is rare. We present a patient with positive urinary antigens for both Streptococcus pneumoniae and Legionella pneumophilia serogroup 1, indicating an unusual co-infection.
\end{abstract}

Categories: Infectious Disease, Pulmonology

Keywords: community-acquired pneumonia, streptococcus pneumonia, legionella pneumophilia, co-infection, urinary antigens

\section{Introduction}

Community-acquired pneumonia (CAP) is a frequent cause of hospitalization in adults leading to significant morbidity and mortality [1]. Most cases of bacterial CAP are caused by a single pathogen, with Streptococcus pneumoniae (S. pneumoniae) identified most commonly [2]. Alternatively, Legionella pneumophila ( $L$. pneumophila) is a less common cause of CAP [2-5]. Although co-infections of various bacterial and viral microorganisms have been described, the presence of both S. pneumoniae and L. pneumophila together in CAP is rare $[2,6]$. We describe a patient with CAP diagnosed with positive urinary antigens for both $S$. pneumoniae and L. pneumophila serogroup 1 (Lp1), indicating an unusual co-infection. A part of this article was presented at the American College of Chest Physicians' annual meeting, CHEST 2017, in Toronto, Canada.

Received 01/01/2019

Review began 02/08/2019 Review ended 02/12/2019 Published 02/16/2019

\section{() Copyright 2019}

Beg et al. This is an open access article distributed under the terms of the Creative Commons Attribution License CC-BY 3.0., which permits unrestricted use, distribution, and reproduction in any medium, provided the original author and source are credited.

\section{Case Presentation}

An 80-year-old Caucasian female who was an active smoker with a 40 pack year smoking history and a past medical history of primary hypertension and chronic obstructive pulmonary disease (COPD) presented to our hospital emergency department (ED) in February, 2017 with fatigue, generalized weakness, and shortness of breath for the last two days. She had been noticing progressively worsening shortness of breath on exertion without any fever, chills, cough, chest pain, orthopnea or paroxysmal nocturnal dyspnea. She did not have any sick contacts and had not travelled anywhere recently.

Upon arrival to the hospital, she was noted to have a heart rate of 122 beats per minute, a respiratory rate of 30 breaths per minute with an oxygen saturation of $85 \%$ on room air, and a blood pressure of $161 / 86 \mathrm{mmHg}$. Further physical examination revealed a thin and cachectic female who appeared to be in mild respiratory distress. She was noted to have normal heart sounds without any murmurs, rubs, or gallops. A pulmonary examination revealed expiratory wheezing bilaterally without any rales or rhonchi. She did not have rashes or peripheral edema. Due to her respiratory distress she was started on non-invasive ventilatory support in the emergency department (ED), which led to improvement in her respiratory status.

Laboratory investigation revealed a hemoglobin count of $15.1 \mathrm{~g} / \mathrm{dL}$ (reference range [ref], 12.3-15.3), a peripheral white blood cell count of 21,130 cells/mm3 (ref, 4400-11,300) with a relative neutrophil percentage of $80 \%$ (ref, 37\%-77\%), a platelet count of 301,000/mm3 (ref, 145,000-445,000), a sodium level of $134 \mathrm{mmol} / \mathrm{L}$, a serum creatinine level of $0.30 \mathrm{mg} / \mathrm{dL}$ (ref, $0.70-1.5$ ), and a blood urea nitrogen of $8 \mathrm{mg} / \mathrm{dL}$ (ref, 9-20). Her liver enzymes were within normal limits. Venous blood gas analysis revealed a $\mathrm{pH}$ of 7.26 and a $\mathrm{CO} 2$ of $83 \mathrm{mmHg}$ (ref, 41-51). Initial serum procalcitonin was $0.07 \mathrm{ng} / \mathrm{mL}$ and a chest radiograph was only significant for hyper-inflated lungs (Figure 1). 


\section{Cureus}

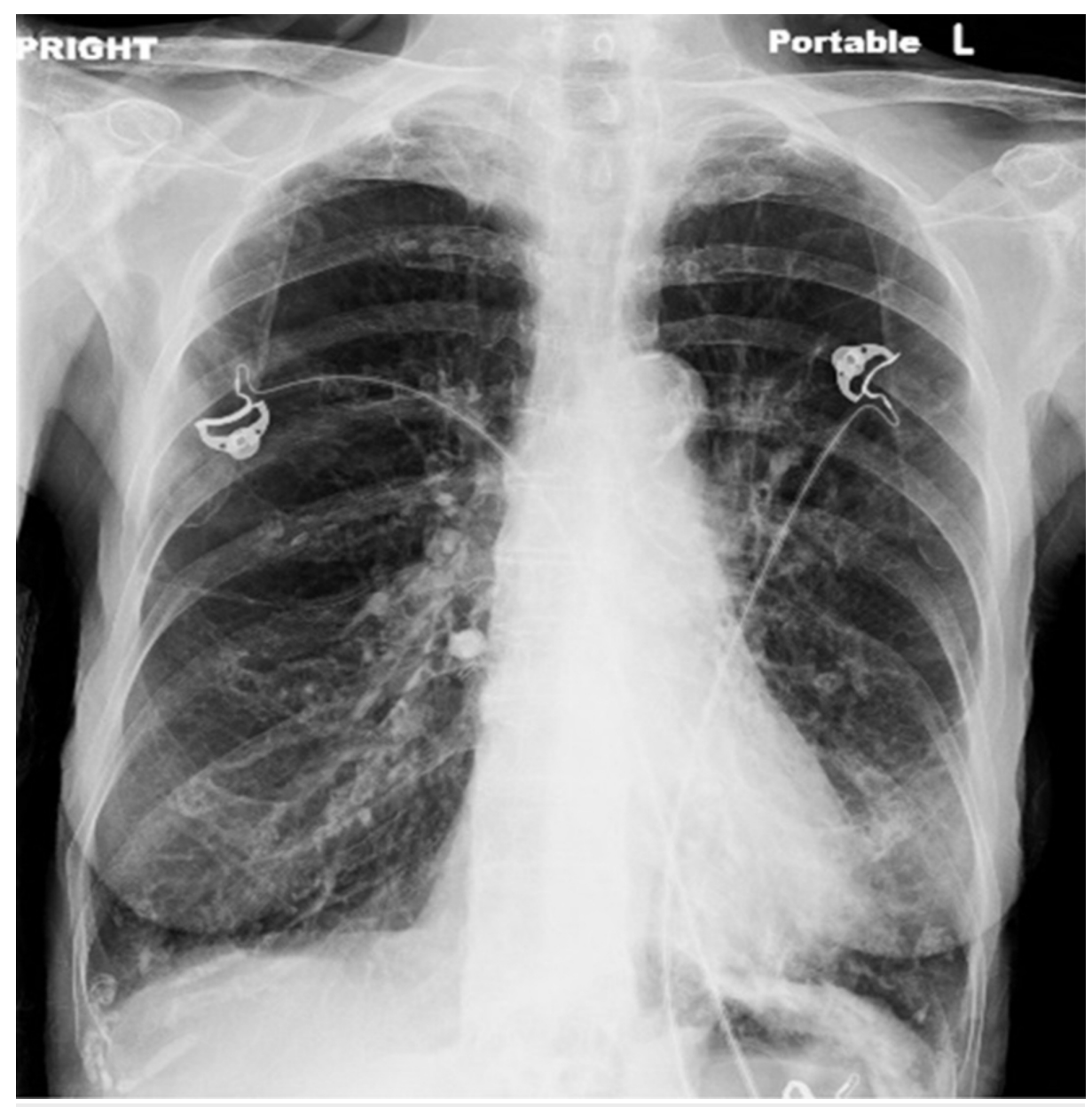

FIGURE 1: Chest X-ray revealing hyper-inflated lungs without any focal consolidation.

She was admitted to the hospital for management of mild COPD exacerbation and treated with inhaled bronchodilators and systemic steroids. Repeat blood work obtained 12 hours after initial presentation was significant for a serum procalcitonin of $2.07 \mathrm{ng} / \mathrm{mL}$ and a peripheral white blood cell count of 35,170 cells $/ \mathrm{mm} 3$ with a relative neutrophil percentage of $91 \%$ and $2 \%$ bands. Due to concerns for development of CAP, sputum cultures, two sets of blood cultures via peripheral blood draw and urinary antigens for $S$. pneumoniae and $L$. pneumophila were obtained. She was started on intravenous ampicillin-sulbactam 3 grams every six hours and azithromycin 500 milligrams every 24 hours. A computed tomography scan of the chest revealed tree-in-bud opacities along with regions of bronchial wall thickening in the bilateral lower lobes (Figure 2). 


\section{Cureus}

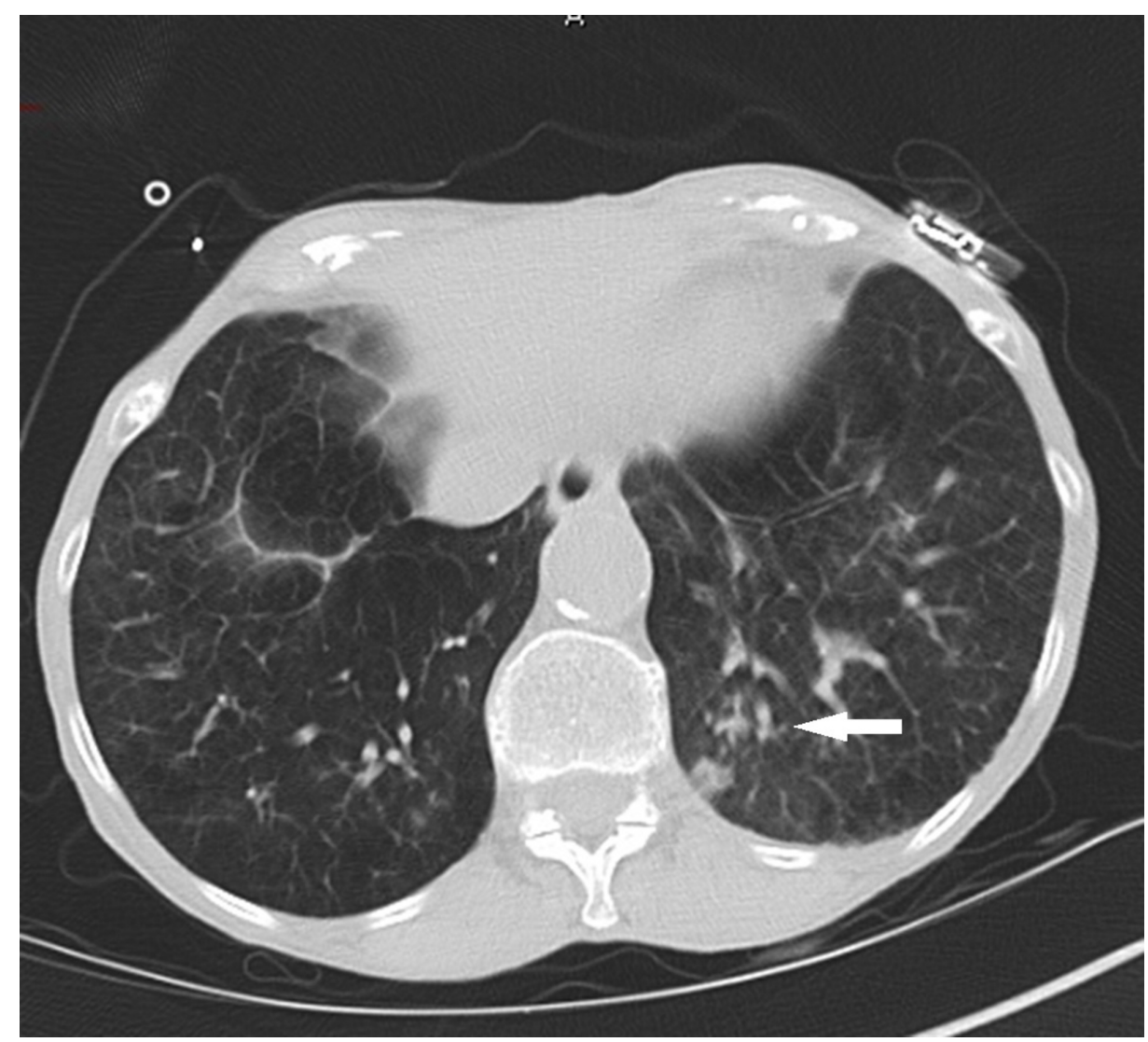

FIGURE 2: Computed tomography of the chest showed tree-in-bud opacities along with bronchial wall thickening (arrow) consistent with an infectious process.

A sputum Gram stain revealed many Gram-positive rods, rare Gram-positive cocci, less than 10 squamous epithelial cells and greater than 25 white blood cells per low power field. Her blood cultures remained negative and the final sputum culture grew normal respiratory flora. Surprisingly, urinary antigens for both S. pneumoniae and Lp1 returned positive. Antibiotics were then de-escalated to intravenous levofloxacin 500 $\mathrm{mg}$ every 24 hours for a total of five days. The patient's clinical status improved, and she was discharged home in a stable condition after a one-week hospital stay.

\section{Discussion}

Although co-infections with S. pneumoniae and L. pneumophila have been described, this appears to be only the second case where a CAP co-infection was diagnosed on the basis of positive urinary antigens [4]. Various studies have shown high specificity and positive predictive value (PPV) of urinary antigens. Thus, our case most likely represents a true co-infection leading to CAP.

One study revealed a specificity of $96 \%$, a PPV of $95.1 \%$, and a likelihood ratio of 19.9 for pneumococcal urinary antigen [7]. Similarly, urinary antigen testing for legionella is also highly specific (99.1\%-99.5\%) indicating that a positive result in the appropriate clinical setting represents a true infection [8-10]. A systematic analysis of 30 studies revealed a pooled specificity of $99.1 \%$ for legionella urinary antigen [10]. However, legionella urinary antigen only detects Lp1 and thus sputum culture on special media remains the gold standard for diagnosis of legionella infections.

Urinary antigen testing should be routinely utilized to identify etiological agents of CAP due to their excellent specificity, but given the poor sensitivities of these tests, a negative result should not be used to rule out infection caused by $S$. pneumoniae or $L$. pneumophila.

False positive cases have been rarely reported for legionella urinary antigen. One case had exposure to rabbit serum antibodies and the other patient had pneumonia and a brain lesion from Nocardia asteroides $[11,12]$. In our patient, although a false positive result is a possibility as the sputum culture for legionella was not performed, she likely had true CAP with legionella and pneumococcus due to her classic signs and symptoms, high specificity of urinary antigen testing, as well as rapid response to antibiotics. 


\section{Conclusions}

This unique case of $S$. pneumoniae and L. pneumophila co-infection demonstrates the importance of urinary antigen testing in patients hospitalized secondary to CAP in addition to obtaining sputum cultures and peripheral blood cultures. This approach will promote early institution of organism-specific antibiotic therapy and foster antibiotic stewardship.

\section{Additional Information \\ Disclosures}

Human subjects: Consent was obtained by all participants in this study. Conflicts of interest: In compliance with the ICMJE uniform disclosure form, all authors declare the following: Payment/services info: All authors have declared that no financial support was received from any organization for the submitted work. Financial relationships: All authors have declared that they have no financial relationships at present or within the previous three years with any organizations that might have an interest in the submitted work. Other relationships: All authors have declared that there are no other relationships or activities that could appear to have influenced the submitted work.

\section{Acknowledgements}

A part of this article was presented at the American College of Chest Physicians' annual meeting, CHEST 2017, in Toronto, Canada.

\section{References}

1. Mandell LA: Community-acquired pneumonia: an overview. Postgrad Med. 2015, 127:607-615. 10.1080/00325481.2015.1074030

2. Jain S, Self WH, Wunderink RG, et al.: Community-acquired pneumonia requiring hospitalization among U.S. adults. N Engl J Med. 2015, 373:415-427. 10.1056/NEJMoa1500245

3. Musher DM, Thorner AR: Community-acquired pneumonia. N Engl J Med. 2014, 371:1619-1628. 10.1056/NEJMra1312885

4. Jover F, Cuadrado JM, Andreu L, et al.: A comparative study of bacteremic and non-bacteremic pneumococcal pneumonia. Eur J Intern Med. 2008, 19:15-21. 10.1016/j.ejim.2007.03.015

5. Beovic B, Bonac B, Kese D, et al.: Aetiology and clinical presentation of mild community-acquired bacterial pneumonia. Eur J Clin Microbiol Infect Dis. 2003, 22:584-591. 10.1007/s10096-003-0997-0

6. Wattanathum A, Chaoprasong C, Nunthapisud P, Chantaratchada S, Limpairojn N, Jatakanon A, Chanthadisai N: Community-acquired pneumonia in southeast Asia: the microbial differences between ambulatory and hospitalized patients. Chest. 2003, 123:1512-1519.

7. Sorde R, Falco V, Lowak M, et al.: Current and potential usefulness of pneumococcal urinary antigen detection in hospitalized patients with community-acquired pneumonia to guide antimicrobial therapy. Arch Intern Med. 2011, 171:166-172. 10.1001/archinternmed.2010.347

8. Reller LB, Weinstein MP, Murdoch DR: Diagnosis of legionella infection. Clin Infect Dis. 2003, 36:64-69. $10.1086 / 345529$

9. Kazandjian D, Chiew R, Gilbert GL: Rapid diagnosis of legionella pneumophila serogroup 1 infection with the binax enzyme immunoassay urinary antigen test. J Clin Microbiol. 1997, 35:954-956.

10. Shimada T, Noguchi Y, Jackson JL, et al.: Systematic review and metaanalysis: urinary antigen tests for legionellosis. Chest. 2009, 136:1576-1585. 10.1378/chest.08-2602

11. Deforges L, Legrand P, Tankovic J, Brun-Buisson C, Lang P, Soussy CJ: Case of false-positive results of the urinary antigen test for legionella pneumophila. Clin Infect Dis. 1999, 29:953-954. 10.1086/520479

12. Bailleul E, Magerman K, Mewis A, Peeters V, Rummens JL, Cartuyvels R: False-positive result with BinaxNOW legionella antigen immunochromatographic (ICT) assay: response to Helbig et al. (2001). J Med Microbiol. 2004, 53:173-0. 10.1099/jmm.0.05000-0 